\title{
Efficient Numerical Integration of Perzyna Viscoplasticity, with Application to Rock Slope Stability using Zero-Thickness Interface Elements
}

\author{
I. Aliguer \& I. Carol \\ Geotechnical Engineering and Geosciences Department, ETSECCPB \\ Universitat Politècnica de Catalunya, Barcelona, Spain
}

\begin{abstract}
In this paper, Perzyna-type viscoplastic rate equations are integrated for a time step by considering the step as stress-driven. Depending on how the increment is imposed (constant, linear etc.), different strategies arise. The secant compliance is obtained by truncated expansion of the yield function. The viscoplastic model can be applied to materials exhibiting rate-dependent behavior, but it can also be used to recover an inviscid elastoplasticity solution when stationary conditions are reached. Within this framework, a viscoplastic relaxation iterative strategy is developed, relating the iterations with the fictitious time steps. Some examples of application are presented in the context of the Finite Element Method with zero-thickness interface elements for slope and stability problems with discontinuities.
\end{abstract}

\section{INTRODUCTION}

Viscoplasticity has been widely used for engineering materials with (physical) time-dependent behaviour over a treshold stress level (Cormeau, 1975, Zienkiewicz and Cormeau, 1974), or in the context of viscoplastic relaxation strategies to obtain the stationary solution of an inviscid problem via a fictitious (non-physical) pseudo-time (Underwood, 1983, Zhang and Yu, 1989). In either case, the rate-type infinitesimal viscoplastic formulation requires a time integration strategy to a) discretize time in increments b) evaluate a linearized relation between stress and strain increments for each time step and, possibly, some residual force calculation and iterative strategy. Typically, the algorithms are based on the initial stress scheme used in FEs, in which the strain increments are prescribed to the constitutive equations. A variety of such algorithms has been proposed since the original constant stiffness and constant stress procedures (Hughes and Taylor, 1978, Peirce et al., 1984, Peric, 1993, Simo, 1991) to more recent and sophisticated contributions (Alfano et al., 2001, Betegon et al., 2006, Lorefice et al., 2008, Ponthot, 2002). In contrast, stress driven schemes are not that common $(\mathrm{Ca}-$ ballero et al., 2009, Zienkiewicz and Cormeau, 1974). Their implementation can be numerically advantageous (explicit integration of the constitutive equations and simple coding) but their stability is strongly related to the size of the time step and, as a consequence, if a small time step is required an enormous amount of iterations are required.

From the viewpoint of the type of viscoplastic formulation there are also differences. While for DuvautLions formulations a good compromise between complexity and cost has been reached via quasi-linear exponential algorithms and the formulation of a consistent viscoplastic tangent operator (Simo and Hughes, 1998, Simo et al., 1988), for Perzyna-type viscoplasticty there seems to be no equivalent approach.

In Rock Mechanics, time-dependency was incorporated in the analysis procedures relatively early, for the overall homogenized behavior of rock masses, and also associated to creep phenomena or salt formations (Winkel et al., 1972, Zienkiewicz and Pande, 1977). In this paper, the continuum is considered as linearelastic, and the non-linearities are restricted to viscoplastic behavior of the rock discontinuities, which are explicitly modeled using zero-thickness interface elements.

\section{CLASSICAL PERZYNA-TYPE VISCOPLASTICITY}

Within the classical framework of small strain Perzyna viscoplasticity Perzyna (1966), total strain can be additively split into the elastic and the viscoplastic:

$\epsilon_{i j}=\epsilon_{i j}^{e l}+\epsilon_{i j}^{v p}$ 
The elastic strain tensor is obtained by considering isotropic linear elasticity:

$\epsilon_{i j}^{e l}=C_{0 i j k l} \sigma_{k l}, \quad C_{0 i j k l}=D_{0 i j k l}^{-1}$

where $\boldsymbol{D}_{0}$ is a elasticity stiffness matrix, symmetric and positive definite. $\boldsymbol{D}_{0}^{-1}$ denotes its inverse, i.e. the elasticity compliance matrix, which will be referred to as $\boldsymbol{C}_{0}$, and $\sigma_{i j}$ is the Cauchy stress tensor.

The stress loading function $F\left(\sigma_{i j}\right)$ is defined to distinguish between elastic states $(F \leq 0)$ and viscoplastic states $(F>0)$. In the latter case, the classical Perzyna viscoplastic strain rate is considered:

$\dot{\epsilon}_{i j}^{v p}=\frac{1}{\eta}\left\langle\psi\left(\frac{F(\boldsymbol{\sigma})}{F_{0}}\right)\right\rangle \frac{\partial Q}{\partial \sigma_{i j}}$

where $\eta$ is the viscosity of the material, $F_{0}$ is a reference value of the yield function for normalization, and $Q$ the viscoplastic potential typical of non-associated formulations.

Finally, the accumulated viscoplastic strain $\epsilon_{i j}^{v p}$ in (1) can be obtained by integrating in time the viscoplastic strain rate:

$\epsilon_{i j}^{v p}=\int_{0}^{t} \dot{\epsilon}_{i j}^{v p} d t=\int_{0}^{t} \frac{1}{\eta}\left\langle\left(\frac{F(\boldsymbol{\sigma})}{F_{0}}\right)\right\rangle \frac{\partial Q}{\partial \sigma_{i j}} d t$

\section{STRESS-DRIVEN NUMERICAL SCHEMES FOR VISCOPLASTIC TIME-INTEGRATION}

As the main difference to traditional elastoplasticity, in viscoplasticity stress states are allowed outside the loading surface. The proposed scheme takes advantage of this fact to obtain a simple but effective algorithm for the integration of Perzyna viscoplasticity. The main distinctive feature of the proposed scheme is that it is stress-driven, in contrast to traditional strain-prescribed procedures for elastoplasticity.

The main advantage of a stress-prescribed scheme is that the integration of the constitutive relation can be reduced to the numerical calculation of the integral expression (4), which becomes relatively simple. In this expression, all the terms are computed in advance, in order to update the current value of the viscoplastic strain, instead of the usual implicit procedures resulting from strain-prescribed schemes.

The integration of the expression (4) can be done via the trapezoid rule in the interval $\Delta t_{n+1}=t_{n+1}-$ $t_{n}$. Depending on the assumption of the stress increment within the interval the following expression for the viscoplastic strain increment can be obtained:

$\Delta \boldsymbol{\epsilon}_{n+1}^{v p}=\frac{\Delta t_{n+1}}{\eta F_{0}}((1-\theta) F(\boldsymbol{\sigma})+\theta F(\boldsymbol{\sigma}+\Delta \boldsymbol{\sigma})) \boldsymbol{m}$

where $\theta$ is a fixed parameter with value between 0 and 1 and $\boldsymbol{m}$ is the flow rule. For $\theta=0$ the original viscoplastic algorithm of Zienkiewicz and Cormeau
Zienkiewicz and Cormeau (1974) with constant stiffness is recovered. For other values of $\theta$ greater than zero, the calculation of $\Delta \boldsymbol{\epsilon}_{n+1}^{v p}$ involves $\Delta \boldsymbol{\sigma}_{n+1}$, and therefore it is required to iterate within the time step until the viscoplastic strain estimates satisfy the prescribed stress increment.

In the implementation developed, a first order expansion of the yield function and the plastic potential is proposed to evaluate the expression above, which leads to a linearized form of $\Delta \epsilon_{n+1}$ in terms of $\Delta \boldsymbol{\sigma}_{n+1}$, as described in more detail in Aliguer et al. (2013).

\section{VISCOPLASTIC RELAXATION}

If a time-dependent problem exhibits stationary conditions, those are characterized by no change in strains. In terms of stress and for the problem at hand, this can be interpreted as stress states evolving from the viscoplastic region $\left(F\left(\sigma_{i j}\right)>0\right)$ to the yield surface/elastic region $\left(F\left(\sigma_{i j}\right) \leq 0\right.$ or stationary state). The viscoplastic relaxation technique takes advantage of this concept, in order to reach the solution of the inviscid problem as the stationary solution of a fictitious viscoplastic problem.

The resulting scheme can be summarized as follows: in the iteration, the viscoplastic strain increment is calculated using only the value of the stress at the beginning of the increment, $\boldsymbol{\sigma}_{n}^{i}$ and the secant stiffness is calculated if the value of $\theta$ is different than 0 . Once the system of equations is solved for $\delta \mathbf{u}_{n+1}^{i}$, the increment of stress for the iteration $\left(\Delta \boldsymbol{\sigma}_{n+1}^{i}\right)$ can be obtained. The iterative procedure reaches convergence when the stress state is sufficiently close to the yield surface, so that the value of residual stress is inferior than a tolerance value.

\section{CONSTITUTIVE MODEL FOR INTERFACES}

The above schemes for viscoplasticity and viscoplastic relaxation have been applied to the simplified version of an existing zero-thickness interface constitutive model, which was previously developed within the context of quasi-static elastoplasticity Caballero et al. (2008) .

The frictional hyperbolic loading function for the interface is defined in terms of normal and shear stresses $\left(\sigma, \tau_{1}, \tau_{2}\right)$ and the strength parameters cohesion and friction angle $(c, \phi)$

$$
F=-\tan \phi(a-\sigma)+\sqrt{\tau_{1}^{2}+\tau_{2}^{2}+a^{2} \tan ^{2} \phi}
$$

where $a=c / \tan \phi$.

The flow rule considered in this model is nonassociative and depends on whether normal stress state is tension or compression. In the case of the tension, the flow rule is radial towards a point near the 
origin, while in compression, only shear viscoplastic strains are generated:

$\mathbf{m}^{\text {tens }}=\left(\begin{array}{c}\partial Q / \partial \sigma \\ \partial Q / \partial \tau_{1} \\ \partial Q / \partial \tau_{2}\end{array}\right)=\left(\begin{array}{c}2\left(\sigma-\sigma^{c u t}\right) \\ 2 \tau_{1} \\ 2 \tau_{2}\end{array}\right)$

$\mathbf{m}^{\text {compr }}=\left(\begin{array}{c}0 \\ 2 \tau_{1} \\ 2 \tau_{2}\end{array}\right)$

where $\sigma^{\text {cut }}$ is a value of small compressive stress which acts as the center for the radial flow rule in tension.

\section{NUMERICAL EXAMPLES}

\subsection{Relaxation test at constitutive level}

The first example of application consists of a relaxation test at constitutive level. Normal stresses are applied first, followed by prescribed shear relative displacement (at constant normal stress). Then time is allowed to pass, and shear stress starts decreasing to the limit value dictated by the yield surface (Fig. 1).
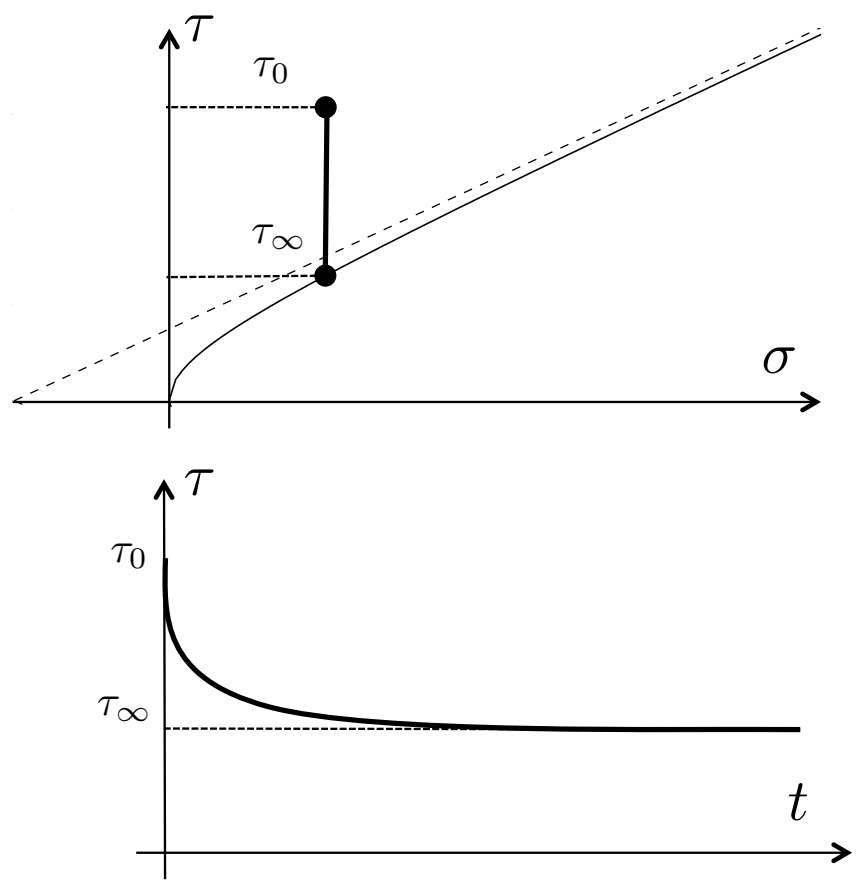

Figure 1: Shear stress path in $\sigma-\tau$ space and shear stress evolution through time

First, the case without cohesion is considered $(c=$ 0 in equation 6). For this particular case, it is possible to find a closed-form expression for the decay of the shear stress with time $\tau=f(t)$ :

$\left(\frac{\tau-\tau_{Y}}{\tau_{0}-\tau_{Y}}\right)=\exp \left(\frac{-K_{T}}{\eta F_{0}} t\right)$

where $\tau_{0}$ is the value of shear stress at time $t_{0}, \tau_{Y}$ is the shear yield threshold value, and $K_{T}$ is the transversal stiffness modulus of the interface. Expression (9) indicates that, at the limit of infinite time, the value $\tau-\tau_{Y}$ tends to zero, that is, the shear stress tends to the shear stress yield limit value $\tau_{Y}$, and the elastoplastic solution is recovered. The resulting curve has been represented in Fig. 2, together with the numerical results for the same case obtained with a $\Delta t=1 \mathrm{~s}$, and three different values of parameter $\theta$ equal to 0,1 and $1 / 2$.

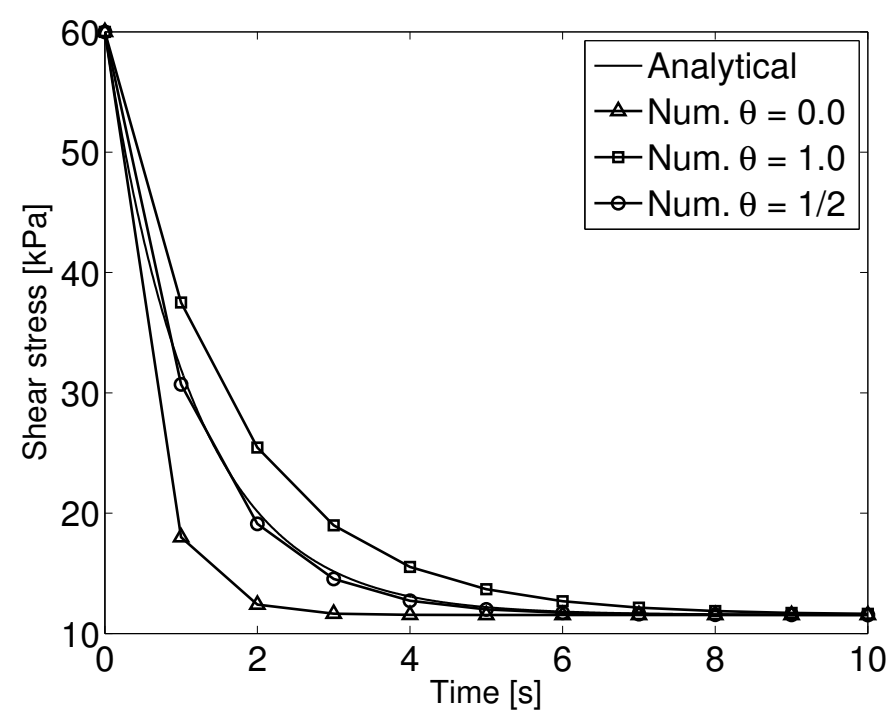

Figure 2: Analytical and numerical results for the evolution of the shear stress with time for $\Delta t=1 \mathrm{~s}$

In the numerical calculations, the load has been applied in two steps: 1) $\Delta u=-2 \cdot 10^{-6} \mathrm{~m}$ and $\Delta v=6 \cdot 10^{-6} \mathrm{~m}$ with $\Delta t=0 \mathrm{~s}$ to generate the initial stress state beyond the yield surface. 2) $\Delta u=\Delta v=0$ and $\Delta t>0$. The second step has been subdivided in 10 increments to reach the final time of $t_{f}=10 \mathrm{~s}$ $(\Delta t=1 s)$. Material parameters used for this example are: $K_{N}=K_{T}=10^{7} \mathrm{kN} / \mathrm{m} ; c=0.0 \mathrm{kN} / \mathrm{m}^{2}$; $\tan \phi=0.577 ; \eta=10^{6} k P a s$.

The figure shows that the strategy with $\theta=1 / 2$ yields a much better approximation than $\theta=0$ or $\theta=1$. A measure of the overall error has been established as the difference of the area under the curves to the one corresponding to the analytical solution, as the total amount of viscoplastic strain will be approximately proportional to that area (equation 3 ). The results obtained give an error lower than $1 \%$ for the strategy with $\theta=1 / 2$, and $14.1 \%$ and $14.0 \%$ for $\theta=0$ or $\theta=1$ respectively. As it would be expected, assuming constant stress for the increment equal to the initial value (forward scheme with $\theta=0$ ), leads to an over-prediction of the viscoplastic strain, and therefore, stress decreases faster. However, assuming $\theta=1$, that is, a backward scheme taking stress at the end of the increment, normally lower, leads to an under-prediction of the viscoplastic strain.

A second calculation has been run with $c=$ $10.0 \mathrm{kN} / \mathrm{m}^{2}$. In this case the analytical solution is not straightforward, and the numerical solution obtained for very small steps of $\Delta t=0.001 \mathrm{~s}$ which leads to 
practically the same results for any $\theta$, is taken as the "exact" solution. This "exact" solution is represented in Fig. 3 together with the numerical results obtained with $\theta=0, \theta=1 / 2$ and $\theta=1$, first for $\Delta t=1 s$ (left diagram), and then for $\Delta t=2.0 \mathrm{~s}$ and $\Delta t=0.5 \mathrm{~s}$ (right diagram).
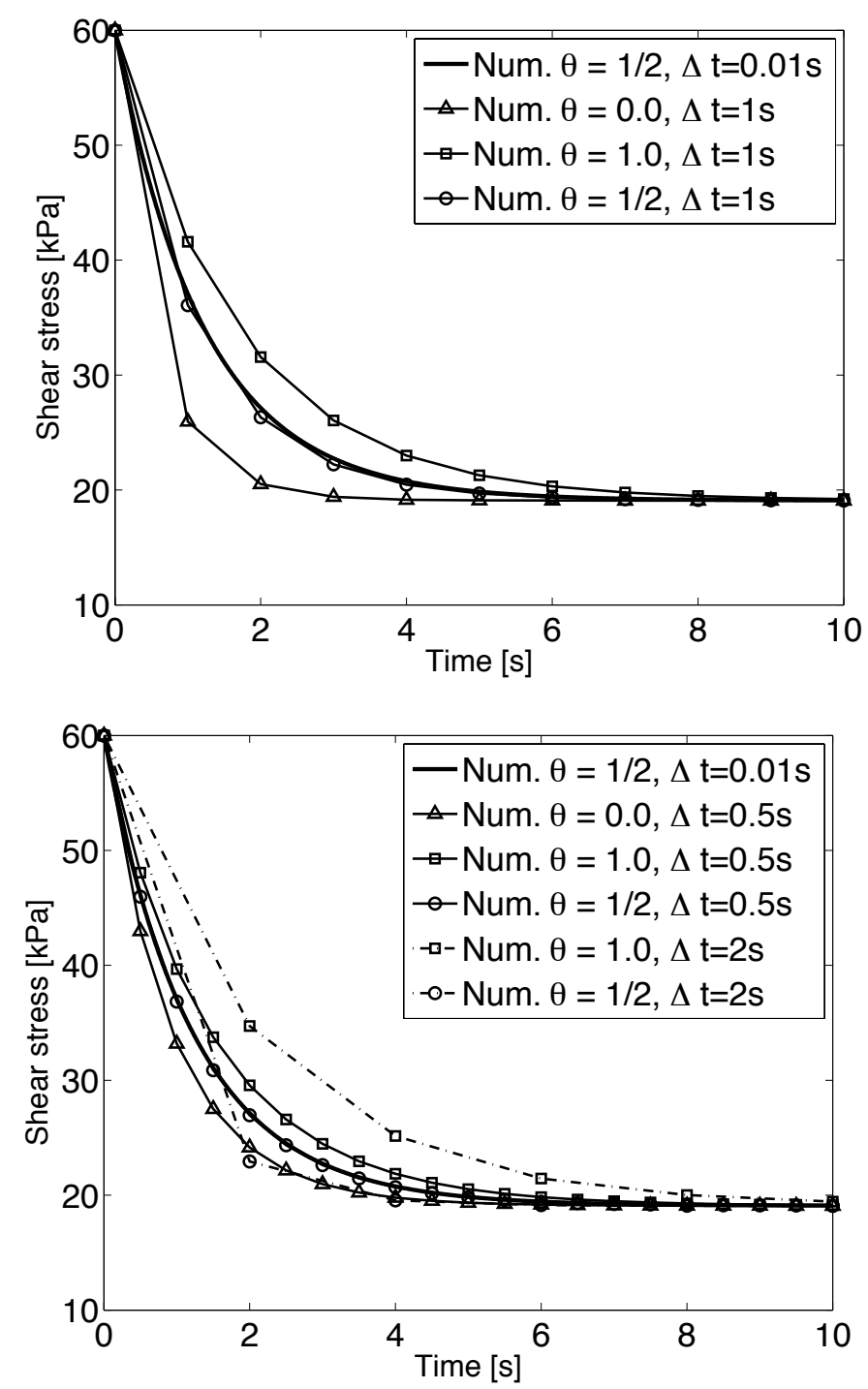

Figure 3: Numerical results for the evolution of the shear stress with time for $\Delta t=1 \mathrm{~s}$ (upper) and $\Delta t=0.5 \mathrm{~s}$ and $\Delta t=2 \mathrm{~s}$ (lower).

Fig. 3 (left) shows a similar trend as Fig. 2. Fig. 3 (right) shows some additional features of the integration schemes, confirming the better performance of $\theta=1 / 2$ and showing that for larger time increments $(\Delta t=2 s)$ convergence is not even obtained for $\theta=0$. The overall error of the three solutions in terms of area under the curves for $\Delta t=0.5 \mathrm{~s}$ are: less than $0.1 \%$ for $\theta=1 / 2$ in front of $4.4 \%$ and $4.3 \%$ for $\theta=0$ and $\theta=1$, respectively. Additionally, calculations have been also run for $\Delta t=0.2 \mathrm{~s}$ with errors of $1.7 \%$ for both $\theta=0$ and $\theta=1$. And one has to decrease $\Delta t$ to the value of $\Delta t=0.05 \mathrm{~s}$ to obtain errors of less than $1 \%$ for those values of $\theta$. This means that for those strategies $(\theta=0$ and $\theta=1)$ one requires about 20 times more time steps to obtain similar accuracy as for $\theta=1 / 2$. Also remarkable is the observation that the backward scheme $\theta=1$ (usually assumed to be more accurate) leads to similar errors as the forward scheme with $\theta=0$.

\subsection{Rock slope stability with zero-thickness interface elements}

The main objective of this example is to evaluate the performance of the viscoplastic relaxation iterative procedure for a classic rock slope analysis (Fig. 4). The following geometry has been considered: $L=$ $100 \mathrm{~m}, H=80 \mathrm{~m}, \Delta p=1.0 \mathrm{kPa} / \mathrm{m}, \beta_{1}=55.1^{\circ}$ and $\beta_{2}=55.7^{\circ}$. For the inclined interfaces the strength parameters used are $c=75 \mathrm{kPa}, \tan \phi=1.0$ and $\eta=$ $10^{6} \mathrm{kPa} \mathrm{s}$, while for vertical interfaces $c=1.0 \mathrm{kPa}$, $\tan \phi=1.4$ and $\eta=10^{6} \mathrm{kPas}$. Rock mass is assumed linear elastic with $E=2 G P a$ and $\nu=0.2$.

First, the initial stress state due to gravity with $K_{0}=0.8$ was generated using the viscoplastic relaxation scheme and $\theta=1 / 2$. Then, the distributed load $\Delta p$ was applied incrementally using a viscoplastic relaxation iterative scheme (also with $\theta=1 / 2$ ) until the failure was reached.

As it can be observed in Fig. 4 different compatible failure mechanisms exist and it is not trivial to determine a priori which is the most unfavorable one. Failure was reached after a total of 103 increments of $\Delta p=1.0 \mathrm{kPa} / \mathrm{m}$, and the failure mechanism was found to be a combination of the first vertical and the lowest inclined joints.

\section{CONCLUDING REMARKS}

An efficient integration procedure for Perzyna-type viscoplasticity with a stress-prescribed scheme has been presented, which can be used for a physical (real time) time-dependent problems or as a basis for a viscoplastic relaxation procedure (fictitious time). The latter allows to recover the elastoplastic solution as a limit inviscid case.

The viscoplastic scheme is successfully implemented for a zero-thickness interface model originally conceived for time-independent representations of rock discontinuities

A constitutive example shows that different values of the parameter $\theta$ lead to different decay curves of shear stress with time, and subsequently also to different total values of accumulated viscoplastic strain. While for $\theta=0$ viscoplastic strains are underestimated, for $\theta=1$ an over-estimation is produced. Best results compared to "exact" solution are obtained with $\theta=1 / 2$.

In the context of the Finite Elements calculation of a rock slope stability problem, the proposed viscoplastic relaxation method is shown capable of detecting the non-trivial failure mechanisms in fractured rock masses. 

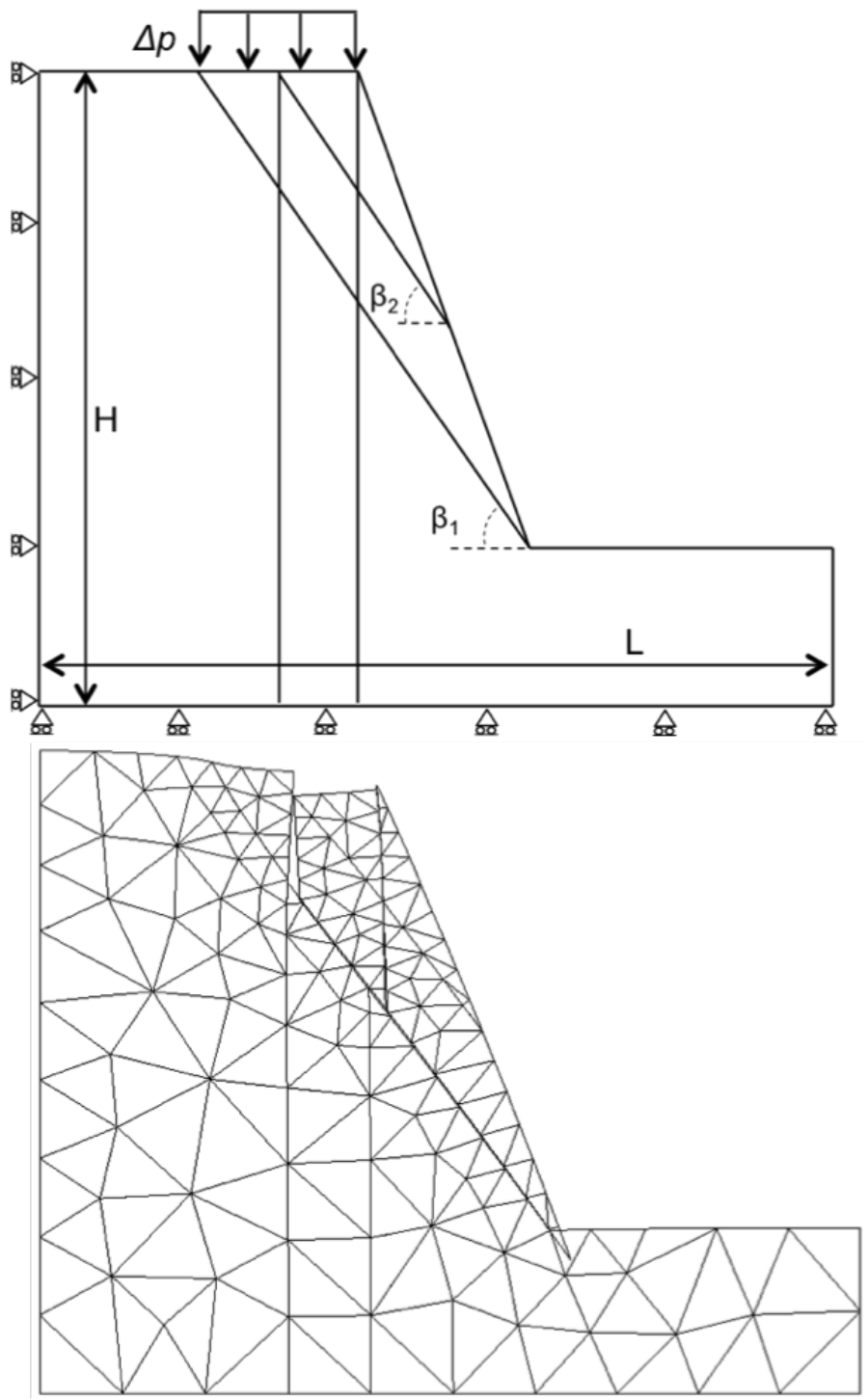

Figure 4: Original geometry and boundary conditions and deformed mesh (x100).

\section{ACKNOWLEDGMENTS}

Partial support for this research has come from project BIA-2012 36898 MEC (Madrid), which includes FEDER funds (European Comission). Funding from 2009SGR-180 from Generalitat de Catalunya (Barcelona) is also greatly appreciated. The first author also wishes to thank MEC (Madrid) for the doctoral fellowship received 2009-2012 including funds for a 6 months stay at CU-Boulder.

\section{REFERENCES}

G. Alfano, F. De Angelis, and L. Rosati. General solution procedures in elasto/viscoplasticity. Computer Methods in Applied Mechanics and Engineering, 190(39):5123 - 5147, 2001.

I. Aliguer, I. Carol, and S. Sture. Stress-driven integration strategies for perzyna-type elasto / viscoplasticity and viscoplastic relaxation. submitted for publication, 2013.

C. Betegon, J.J. del Coz, and I. Penuelas. Implicit integration procedure for viscoplastic gurson materials. Computer Methods in Applied Mechanics and Engineering, 195(44-47):6146-6157, 2006.

A. Caballero, K.J. Willam, and I. Carol. Consistent tangent formulation for 3D interface modeling of cracking/fracture in quasi-brittle materials. Computer Methods in Applied Mechanics and Engineering, 197(33-40):2804 - 2822, 2008.

A. Caballero, D. Garolera, I. Carol, and A. Gens. Viscoplastic multilaminate model for jointed rock with stress-prescribed using a stress-prescribed LEGI scheme. In Proceedings of the Int. Conf. on Rock Joints and Jointed Rock Masses, pages 1-8, Tucson, AZ, 2009.

I.C. Cormeau. Numerical stability in quasi-static elasto/visco-plasticity. International Journal for Numerical Methods in Engineering, 9(1):109-127, 1975.

T.J.R. Hughes and R.L. Taylor. Unconditionally stable algotiyhms for elastic/ visco-plastic finite element analysis. Computers and Structures, 8(2): 169-173, 1978.

R. Lorefice, G. Etse, and I. Carol. Viscoplastic approach for rate-dependent failure analysis of concrete joints and interfaces. International Journal of Solids and Structures, 45(9):2686-2705, 2008.

D. Peirce, C.F. Shih, and A. Needleman. A tangent modulus method for rate dependent solids. Computers and Structures, 18(5):875 - 887, 1984.

D. Peric. On a class of constitutive equations in viscoplasticity: Formulation and computational issues. International Journal for Numerical Methods in Engineering, 36(8):1365-1393, 1993.

P. Perzyna. Fundamental problems in viscoplasticity. Advances in Applied Mechanics, 9(C):243377, 1966.

J.P. Ponthot. Unified stress update algorithms for the numerical simulation of large deformation elastoplastic and elasto-viscoplastic processes. International Journal of Plasticity, 18(1):91-126, 2002.

J. Simo and T.J.R. Hughes. Computational Inelasticity. Springer, Berlin, 1998.

J. C. Simo, J. G. Kennedy, and S. Govindjee. Nonsmooth multisurface plasticity and viscoplasticity. loading/unloading conditions and numerical algorithms. International Journal for Numerical Methods in Engineering, 26(10):2161-2185, 1988.

J.C. Simo. Nonlinear stability of the time-discrete variational problem of evolution in nonlinear heat 
conduction, plasticity and viscoplasticity. Computer Methods in Applied Mechanics and Engineering, 88(1):111-131, 1991.

P. Underwood. Dynamic Relaxation, volume 1 of Computational Methods for Transient Analysis, pages 246-265. Elsevier, 1983.

B.V. Winkel, K.H. Gerstle, and H.Y. Ko. Analysis of time-dependent deformations of openings in salt media. International Journal of Rock Mechanics and Mining Sciences \& Geomechanics Abstracts, 9 (2):249 - 260, 1972.

L.G. Zhang and T.X. Yu. Modified adaptive dynamic relaxation method and its application to elasticplastic bending and wrinkling of circular plates. Computers and Structures, 33(2):609-614, 1989.

O.C. Zienkiewicz and I.C. Cormeau. Visco-plasticity - plasticity and creep in elastic solids - a unified numerical solution approach. Int. J . Numer. Meth. Engng., 8(4):821 - 845, 1974.

O.C. Zienkiewicz and G. N. Pande. Time-dependent multilaminate model of rocks a numerical study of deformation and failure of rock masses. International Journal for Numerical and Analytical Methods in Geomechanics, 1(3):219-247, 1977. 